LISA Pathfinder: the experiment and the route to LISA

This article has been downloaded from IOPscience. Please scroll down to see the full text article.

2009 Class. Quantum Grav. 26094001

(http://iopscience.iop.org/0264-9381/26/9/094001)

View the table of contents for this issue, or go to the journal homepage for more

Download details:

IP Address: 194.94.224.254

The article was downloaded on 08/10/2010 at 09:19

Please note that terms and conditions apply. 


\title{
LISA Pathfinder: the experiment and the route to LISA
}

\author{
M Armano $^{1}$, M Benedetti ${ }^{2}$, J Bogenstahl $^{3}$, D Bortoluzzi $^{4}$, P Bosetti $^{4}$, \\ N Brandt ${ }^{5}$, A Cavalleri ${ }^{6}$, G Ciani ${ }^{6}$, I Cristofolini ${ }^{4}$, A M Cruise ${ }^{7}$, \\ K Danzmann ${ }^{8}$, I Diepholz ${ }^{8}$, G Dixon $^{7}$, R Dolesi $^{6}$, J Fauste $^{1}$, L Ferraioli $^{6}$, \\ D Fertin ${ }^{9}$, W Fichter ${ }^{5}$, M Freschi ${ }^{1}$, A García ${ }^{8}$, C García ${ }^{9}$, A Grynagier ${ }^{5}$, \\ F Guzmán $^{8}$, E Fitzsimons ${ }^{3}$, G Heinzel $^{8}$, M Hewitson ${ }^{8}$, D Hollington ${ }^{10}$, \\ J Hough $^{3}$, M Hueller ${ }^{6}$, D Hoyland ${ }^{7}$, O Jennrich ${ }^{9}$, B Johlander ${ }^{9}$, \\ C Killow $^{3}$, A Lobo ${ }^{11}$, D Mance ${ }^{12}$, I Mateos ${ }^{11}$, P W McNamara ${ }^{9}$, \\ A Monsky $^{8}$, D Nicolini ${ }^{9}$, D Nicolodi ${ }^{6}, M_{\text {Nofrarias }}^{8}$, M Perreur-Lloyd $^{3}$, \\ E Plagnol $^{13}$, G D Racca ${ }^{9}$, J Ramos-Castro ${ }^{14}$, D Robertson ${ }^{3}$, J Sanjuan ${ }^{11}$, \\ M O Schulte ${ }^{10}$, D N A Shaul ${ }^{10}$, M Smit $^{15}$, L Stagnaro ${ }^{9}$, F Steier ${ }^{8}$, \\ T J Sumner ${ }^{10}$, N Tateo ${ }^{6}$, D Tombolato ${ }^{6}$, G Vischer ${ }^{8}$, $\mathbf{S}$ Vitale $^{6}$,

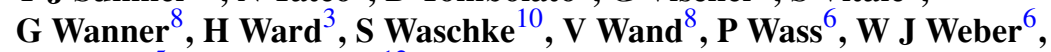 \\ T Ziegler ${ }^{5}$ and $\mathbf{P}$ Zweifel $^{12}$
}

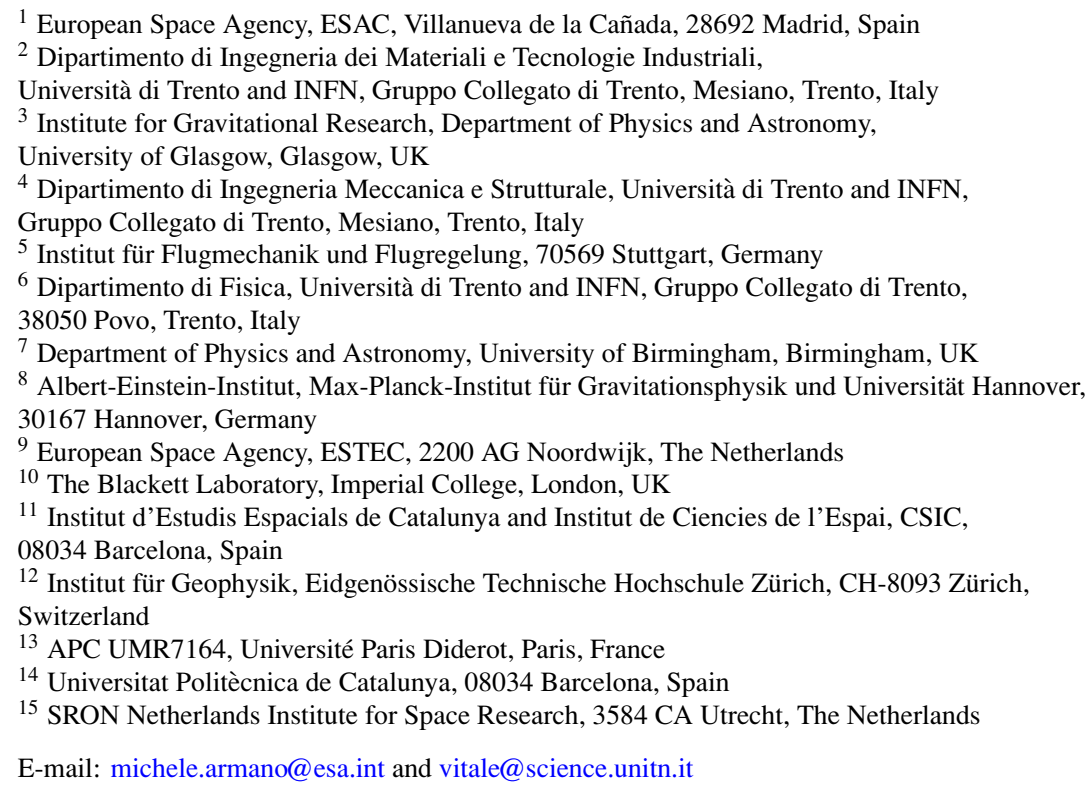

Received 30 October 2008, in final form 21 January 2009

Published 20 April 2009

Online at stacks.iop.org/CQG/26/094001

\section{Abstract}

LISA Pathfinder (LPF) is a science and technology demonstrator planned by the European Space Agency in view of the LISA mission. As a scientific payload, 
the LISA Technology Package on board LPF will be the most precise geodesics explorer flown as of today, both in terms of displacement and acceleration sensitivity. The challenges embodied by LPF make it a unique mission, paving the way towards the space-borne detection of gravitational waves with LISA. This paper summarizes the basics of LPF, and the progress made in preparing its effective implementation in flight. We hereby give an overview of the experiment philosophy and assumptions to carry on the measurement. We report on the mission plan and hardware design advances and on the progress on detailing measurements and operations. Some light will be shed on the related data processing algorithms. In particular, we show how to single out the acceleration noise from the spacecraft motion perturbations, how to account for dynamical deformation parameters distorting the measurement reference and how to decouple the actuation noise via parabolic free flight.

PACS numbers: $04.80 . \mathrm{Nn}, 95.55 . \mathrm{Ym}$

(Some figures in this article are in colour only in the electronic version)

\section{Introduction}

LISA Pathfinder [1-3] (LPF) is a European Space Agency (ESA) mission dedicated to demonstrate the feasibility of the measurement scheme of LISA with ten orders of magnitude reduction in scales but only a relaxation of one-tenth on the required accuracy. At test in LPF is our ability to put observers in free-fall tracking their mutual position, minimizing the experimental disturbances connected to the operation and shielding the observers from local environmental noise.

Ideally, the basic constituent of this scheme is a Doppler link entailing two point particles_-an emitter and a receiver-exchanging an electromagnetic beam whose frequency is measured at reception. Each arm of LISA contains two counter-propagating copies of such a link while LPF flies a downscaled version of it. LISA and LPF share this measurement strategy with other experiments in gravitational physics (one example for all being the deep space radio-science probes), but demand an unprecedented sensitivity and quality of free-fall due to the weak strain of gravity waves LISA targets.

The relative frequency shift between emitter and receiver is $[4,5]$

$$
\frac{\Delta v}{v}=-k_{\mu}\left(x_{r}^{\lambda}\right)\left(v_{r}^{\mu}\left(x_{r}^{\eta}\right)-v_{e}^{\mu}\left(x_{e}^{\eta} \rightarrow x_{r}^{\eta}\right)\right)
$$

Here $k^{\mu}$ is the beam wave 4-vector, $v_{e}^{\mu}$ and $v_{r}^{\mu}$ are the velocity 4-vectors of the receiver and the emitter, respectively. The receiver's velocity is measured at the event of beam reception $x_{r}^{\eta}$. The emitter's one is measured at emission $x_{e}^{\eta}$ and then parallel transported along the light beam to $x_{r}^{\eta}$, this way exploring the intervening gravitational field.

For gravitational signals, the frequency shift in (1) is time varying. In LISA, for instance, the laser frequency shift oscillates at the frequency of the gravitational wave. In radio-science experiments, the curvature of the gravitational field shows up as a rapid variation of the frequency while the radio beam grazes the Sun [6].

Three broad categories of disturbances affect the ideal measurement by causing time variations of the frequency. 

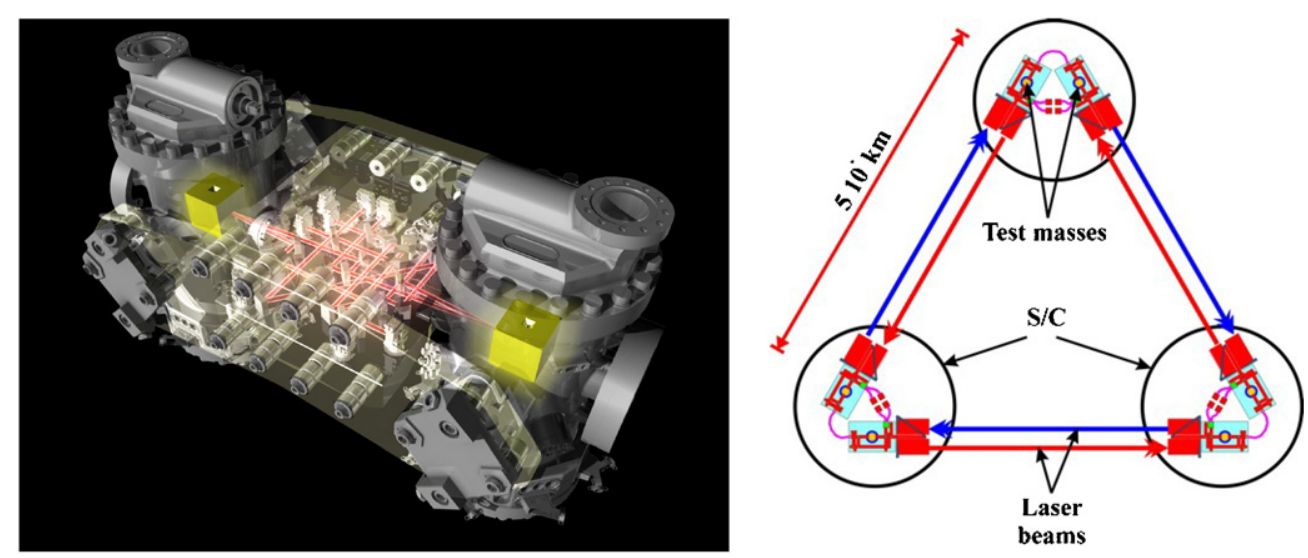

Figure 1. Computer graphics picture of the LTP experiment (left) and LISA constellation schematic (right).

The first is the relative motion of emitter and receiver due to non-gravitational forces. Considering the (weak) gravity field as a background and to first order in $1 / c$, the time derivative of the frequency shift in equation (1) at the receiver, reads [1]

$$
\frac{\mathrm{d}}{\mathrm{d} t} \frac{\Delta v(t)}{v} \simeq-\frac{1}{c} \Delta a_{e}\left(t-\frac{L}{c}\right) .
$$

In (2) $L$ is the distance travelled by the beam, while $\Delta a_{e}$ is the ordinary acceleration of the emitter in the rest frame of the receiver. The Doppler link behaves then as a time-delayed, differential accelerometer along the line of sight. In the low velocity limit considered here, this differential acceleration contribution just superimposes to the signal as a disturbance.

The second disturbance affecting the measurement is the readout noise. An equivalent acceleration phase noise corrupts LISA's heterodyne interferometer readout signal from the various beams. As the phase needs to be time derived twice to be compared with the frequency time derivative in (2), the phase readout noise power spectrum gets multiplied by the fourth power of the frequency. Whatever the details of the spectrum, this steep frequency dependence makes the readout noise dominating over the acceleration disturbance above some frequency that, for LISA, is expected to be of a few $\mathrm{mHz}$.

The Doppler link constituting the ideal measurement scheme is realized in LISA by a pair of test masses (TMs). Each spacecraft (SC) in LISA will host two TMs, and the sensing interferometric arm will be created shining a laser beam along the $5 \times 10^{6} \mathrm{~km}$ between TMs residing in different SC (see figure 1). The third source of disturbance thus comes from the departure of the actual LISA scheme from the ideal 5 million km link, point-to-point measurement. In reality the measurement is a combination of three different links, two between each TM and its hosting SC and one between the two SC. In addition all these bodies are extended, thus each link measures the relative acceleration of two somewhat arbitrary points on the two linked bodies. Only if all these points are exactly located along the line joining the centres of mass of the TM, the accelerations of the SC and the rotation of the TMs are not leaking into the signal.

The LISA Technology Package (LTP) experiment on LPF aims at delivering an experimentally tested physical model for all three categories of disturbances above, with an accuracy sufficient to quantitatively predict LISA performance. LPF top science goal is qualifying the acceleration noise in a space environment mimicking that of LISA up to 7 times 
worse than LISA sensitivity threshold. Indicating the power spectral density (PSD) of $\Delta a_{\mathrm{e}}$ with $S_{\triangle a_{\mathrm{e}}}$, the performance baseline of LISA can be written as

$S_{\Delta a}^{1 / 2} \leqslant \sqrt{2} \times 3 \times 10^{-15} \sqrt{1+\left(\frac{f}{3 \mathrm{mHz}}\right)^{4}} \mathrm{~ms}^{-2} \mathrm{~Hz}^{-1 / 2}, \quad f \geqslant 0.1 \mathrm{mHz}$.

As downscaled version of the link, the LTP is entirely contained within one spacecraft, and using nominal LISA hardware wherever is possible. Despite the downscaling, the experiment covers a large fraction of the relevant physics of the disturbance phenomena described above. LISA's sensing arm is reduced in LTP to $38 \mathrm{~cm}$, with three main effects to depart from the LISA measurement scheme:

(1) the acceleration and displacement noise upon the two TMs will be correlated;

(2) chasing two TMs along a collinear sensing line within the same SC will be impossible and will force to electrostatically suspend one of them (while chasing two TMs along non-parallel sensing lines is challenging but possible for each SC of LISA, see section 3);

(3) as the sensitivity to gravitational waves strain scales like $\delta L / L$, with $L$ being the interferometer armlength, this will make LTP insensitive towards them. LTP will then be a local acceleration difference detector.

Reducing and subtracting the different noise contribution to demonstrate (3) is the key strategy of the LPF mission. This is pursued in two ways:

(1) the experimental hardware is carefully designed to minimize noise excess;

(2) the measurement procedures will explore the noise contributions attributed to different sources (see section 4).

We will expand the first point in section 2 dedicated to the hardware description [2], while the second subject will be tackled in sections 3 and 4. Sections 5, 6 and 7 will explain some measurement procedures in detail.

\section{LISA Pathfinder: hardware and mission}

\subsection{The inertial sensor core}

The TMs will consist of two gold-platinum cubes, weighing $1.96 \mathrm{~kg}$ for a $46 \mathrm{~mm}$ side. Such optimal mono-phasic alloy favours low magnetic susceptibility $\left(\leqslant 10^{-5}\right)$ and maximizes weight due to its high density $\left(\sim 2 \times 10^{4} \mathrm{~kg} \mathrm{~m}^{-3}\right)$, thus reducing the force noise impact on local accelerations (as $\Delta F / m_{\mathrm{TM}} \simeq \Delta a$ ).

Each TM will be hosted inside a sensing electrode housing $(\mathrm{EH})$, a hollow molybdenum structure hosting gold-coated sapphire electrodes (see figure 2). This set of capacitorsattached to the inner EH walls and named gravity reference sensor (GRS) — will track the TMs' motion (displacements and rotations) through the voltage changes induced by the variation of capacitance. The electrodes will also be employed to displace the TMs by applying electrostatic potentials. The signals from/to the electrodes are coupled and controlled by a dedicated front-end-electronics in charge of sensing and actuation. For both these purposes the GRS is designed to minimally perturb the TMs while getting the maximal amount of information on TMs' position: the gaps between TMs and capacitors are $4 \mathrm{~mm}$ to optimize sensitivity to $\leqslant 2 \mathrm{~nm} \mathrm{~Hz}^{-1 / 2}$ and voltages are kept low to reduce the upper limit to sensing stiffness and static gravity gradients to $\leqslant 4 \times 10^{-7} \mathrm{~s}^{-2}$ [7]. Only AC voltage carriers are allowed on the TM in free-fall, banning dc contributions that would induce fluctuating forces at low frequency. Final figures for the GRS comprise a displacement noise of $1.8 \mathrm{~nm} \mathrm{~Hz}^{-1 / 2}$ and attitude noise of $200 \mathrm{~nm} \mathrm{~Hz}^{-1 / 2}$. 


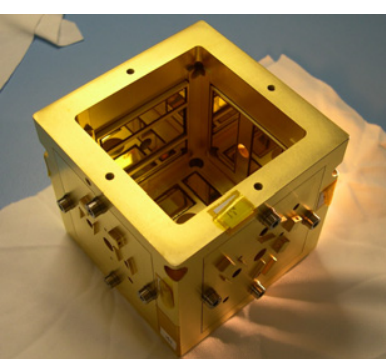

(a)

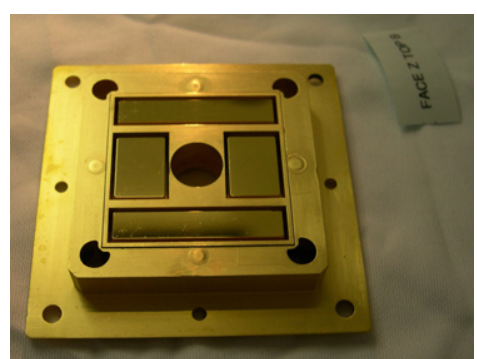

(b)

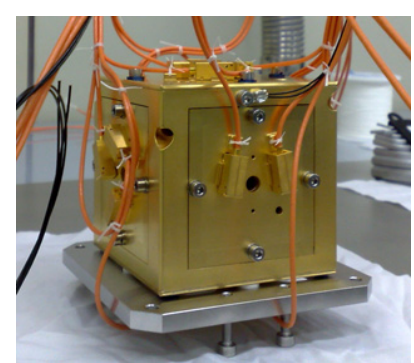

(c)

Figure 2. The EH (a), a detail of the gold-coated sapphire electrodes mounted inside the cap surface $(b)$ and the full assembly with connected wires $(c)$. Pictures courtesy of INFN.

Each EH is embedded in a dedicated vacuum enclosure manufactured from titanium (due to its non-magnetic properties). Its structure and subsystems maintain the EH in deep vacuum, keep the temperature stable and compensate for outgassing and virtual leaks (see figure 5 (left) for a schematic).

\subsection{Interferometry}

Current interferometric technology on ground [8] involves the use of laser tracking with resolution $\leqslant 10^{-18} \mathrm{~m} \mathrm{~Hz}^{-1 / 2}$ at frequencies $>100 \mathrm{~Hz}$. For LPF we rely on the interferometer readout within the measurement bandwidth $(\mathrm{MBW})$ at frequencies around the $\mathrm{mHz}-$ representative of the core of LISA's MBW. The employed Nd:YAG NPRO laser has $35 \mathrm{~mW}$ power and a wavelength of $1064 \mathrm{~nm}$ (see figure 4); light is delivered through a single-mode polarization-maintaining fibre and then split into two paths, each of which is passed through an acousto-optic modulator, driven at $80 \mathrm{MHz}$ and $80 \mathrm{MHz}+2 \mathrm{kHz}$, generating a heterodyne frequency of $2 \mathrm{kHz}$. The beams are delivered to a Zerodur base-plate optical bench, whose silica optics are bonded to the surface employing the technique of hydroxide catalysis [9]. Such a choice of material and manufacturing meets thermal and mechanical requirements ensuring the total metric noise added by the optics not to exceed $10^{-12} \mathrm{~m} \mathrm{~Hz}^{-1 / 2}$ in the MBW [10].

One laser beam is used on the bench as a reference, while the other one is reflected to form six measurement paths, effectively creating four heterodyne interferometers with quadrant photodiode detectors. This allows us to track the relative displacement of the TMs ( $\Delta x$, main science signal), the position of one TM with reference to the bench (and thus position and attitude towards the SC), the laser frequency noise (Mach-Zehnder unequal-arms configuration) and the noise floor reference (rigid equal-arm configuration). The spare two paths are employed to track the laser intensity noise for both reference and measurement beams via photodiodes collecting the light from each fibre (see figure 3 ).

A phase meter assembly samples the signal from each photodiode at $100 \mathrm{~Hz}$. The signals phases are evaluated by an efficient single-bin discrete Fourier transform thus producing TMs' displacement and attitude estimators. Before transmission to the on-board computer, data sets are downsampled to $10 \mathrm{~Hz}$ by a data management unit [11] that will also control the thermometers-heaters and coils-magnetometers assemblies (employed for diagnostics and further acceleration noise characterization) [12]. The final sensitivity of the optical readout will have a detection noise floor of $9 \mathrm{pm} \mathrm{Hz}^{-1 / 2}$ in displacement and of $20 \mathrm{nrad} \mathrm{Hz}^{-1 / 2}$ in attitude. 

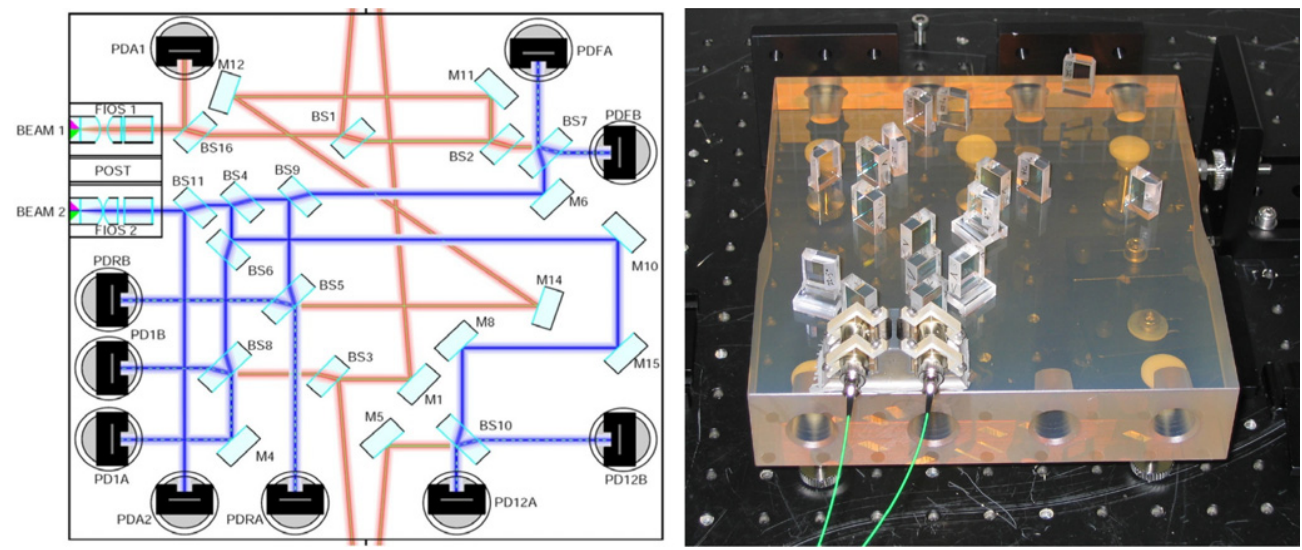

Figure 3. Optical paths (left) between mirrors and optics of the engineering model of the optical bench (right).
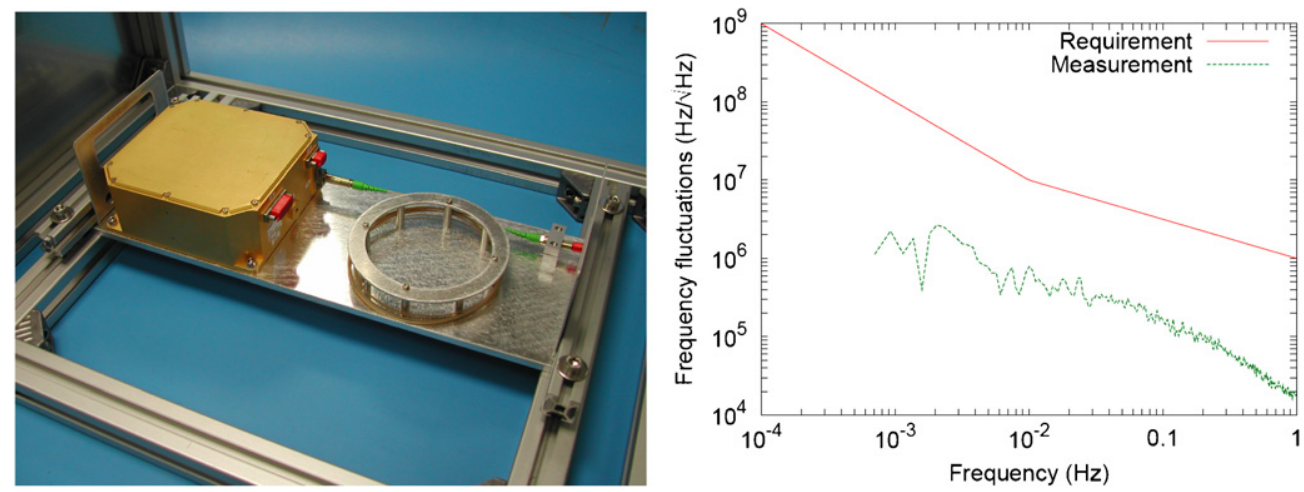

Figure 4. The Nd:YAG NPRO reference laser unit (left) and recent results on laser testing showing a performance in frequency fluctuation at least one order of magnitude better than the requirement (right).

\subsection{Controls}

TM1 will be electrostatically suspended using high-gain control laws to reduce coupling with the SC jitter, TM2 will 'drag-free' [13] and its displacement and attitude signals will be used to steer the SC. The roles of the TMs are interchangeable.

The drag-free and attitude control system (DFACS) [14, 15] will embody the controls needed to realize this scheme. Amongst other duties, the DFACS aims at:

(i) providing a high-gain default mode to suspend and stiffly control both TMs in the case of environmental forces beyond threshold ('accelerometer mode');

(ii) maximizing the experimental time spent by the system in the so-called 'science mode', the optimal drag-free designed to keep the TMs' mutual distance fixed and to reduce jittering of the TM-TM displacement;

(iii) constraining displacement fluctuation between each $\mathrm{TM}$ and the $\mathrm{SC}$ below $5 \mathrm{~nm} \mathrm{~Hz}^{-1 / 2}$; 

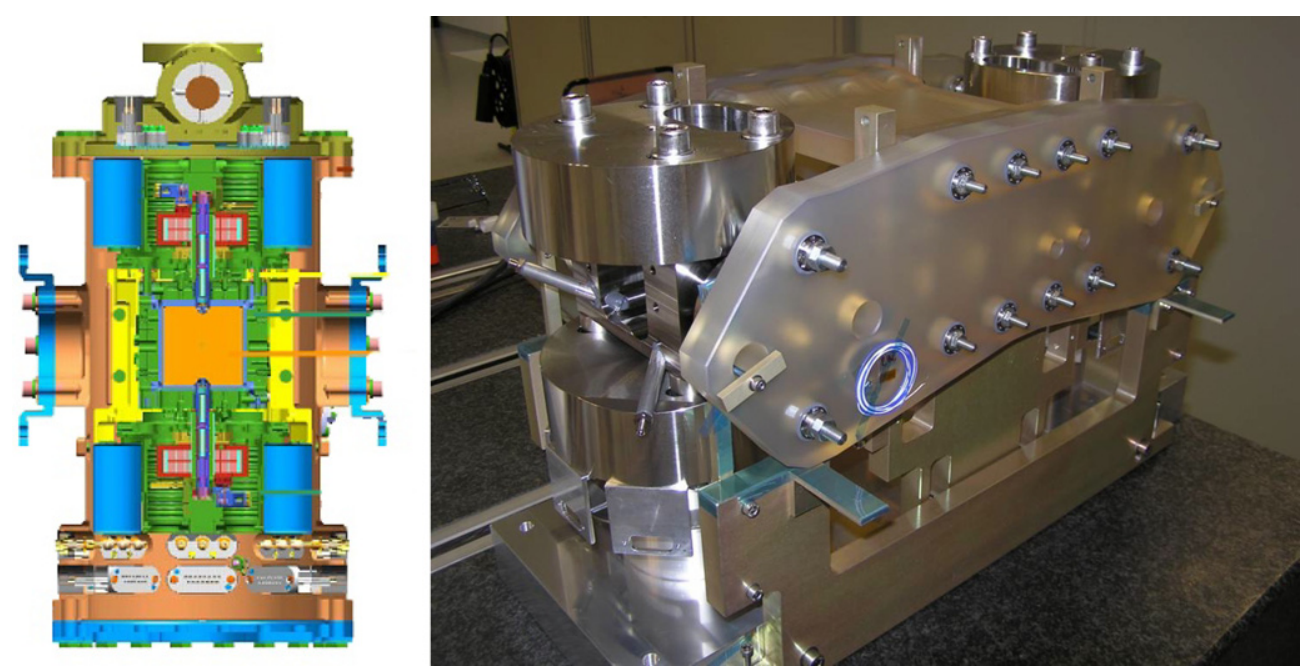

Figure 5. Schematics of one inertial sensor (left) and the mounting frame of the two sensors (right).

(iv) avoiding extra perturbation to disturb the drag-free TM, including direct actuation signals in band, allowing dc forces only to suspend the reference TM;

(v) keeping orthogonality of different d.o.f.'s to minimize cross-talk.

It has been frequently noted that a set of control laws incarnating these rules will effectively amount to keep the TM-SC-TM setup in the most precise formation flight ever built.

\subsection{Charging}

No contact will be allowed between the TMs and the SC: more and more charge will build up on the TMs due to high-energy particles hitting LPF. The scaling factor between lowfrequency commanded rotation and measured attitude of each TM around the $\theta$ d.o.f. will be used as a charge estimator. Electrons will be extracted from the TMs or their EHs by shining their surfaces with UV light generated with mercury lamps and properly routed via optical fibres. The discharge system will operate in a twofold way: a continuous discharge mode active during science activities (low illumination intensity) and a rapid discharge mode, incompatible with science operations (maximum light intensity) [16].

\subsection{Caging}

The extreme acceleration conditions at launch, coupled with the relatively heavy TMs and large gaps in the EHs make it mandatory to equip LPF with a proper lock-release device for the TMs. The random load of $\sim 50 \mathrm{~g}$ RMS at liftoff would heavily damage the TMs and surrounding electrodes. A caging mechanism will keep the TMs in place by applying a $2 \mathrm{kN}$ force, without damaging their outer gold coating and releasing them at need with a displacement precision of $200 \mu \mathrm{m}$ and maximal residual velocity of $5 \times 10^{-6} \mathrm{~m} \mathrm{~s}^{-1}$. The caging device (see figure 6) consists of three actuators of decreasing strength and size: hydraulic, positioning and release. The first to exert the maximum locking force on the TMs' corners via 4 poles per side (for a total of 8 fingers per TM), the second and third forming a telescopic plunger to centre the TMs and set them free by breaking the residual metal surface adhesion. 


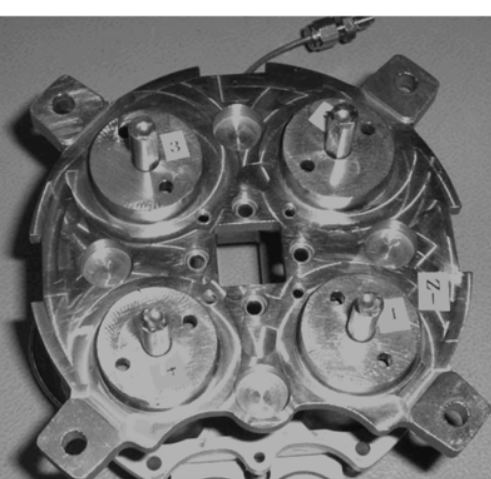

(a)

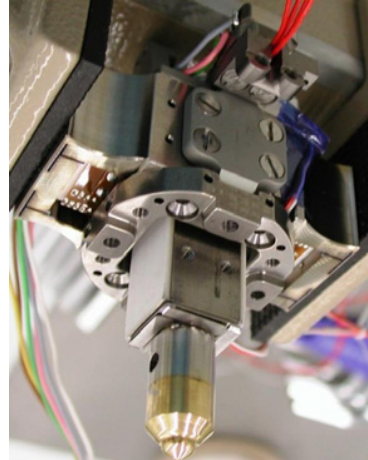

(b)

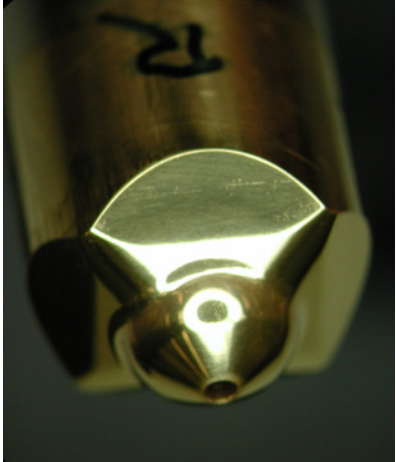

$(c)$

Figure 6. The caging mechanism with its three actuators: (a) hydraulic, $(b)$ positioning and (c) release.
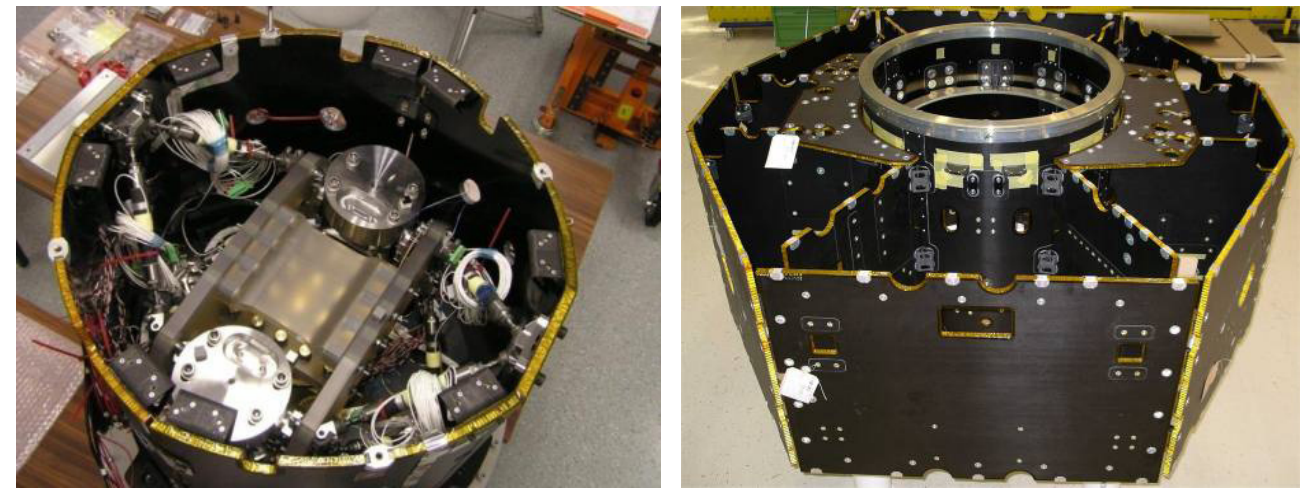

Figure 7. The LTP experiment inside the central hull (left) of the SC and a broader view of the SC octagonal structure (right).

\subsection{Spacecraft and micro-propulsion}

The LPF SC has the shape of an octagonal prism (see figure 7) with structural panels made of CFRP (aluminium cores with carbon fibre laminate skin). The LTP will be accommodated into the central cylindrical area of the SC: a set of struts will sustain the scientific core instrument and reduce the transferred vibrational noise. To guarantee magnetic, electric and static gravitational cleanliness all the SC units are mounted on the inner shear walls between the core cylinder and the outer enclosure (the farthest possible from LTP) maximizing central symmetry. Thermal stability policy involves the use of sensing thermometers, heaters and radiators; the whole module is wrapped in a multi-layer thermal insulation cocoon.

The SC 'absolute' attitude will be read with reference to far-field constellations by star trackers with baseline sensitivity of 32 arcsec. This information will be delivered to the DFACS end employed by low-frequency control laws coupled to the higher-priority SC angular controls based on the GRS TMs' signals.

The SC will be displaced and rotated by means of field emission electric propulsion (FEEPs). FEEPs are micro-propulsion thrusters exerting a pushing force in the $\mu \mathrm{N}$ range 

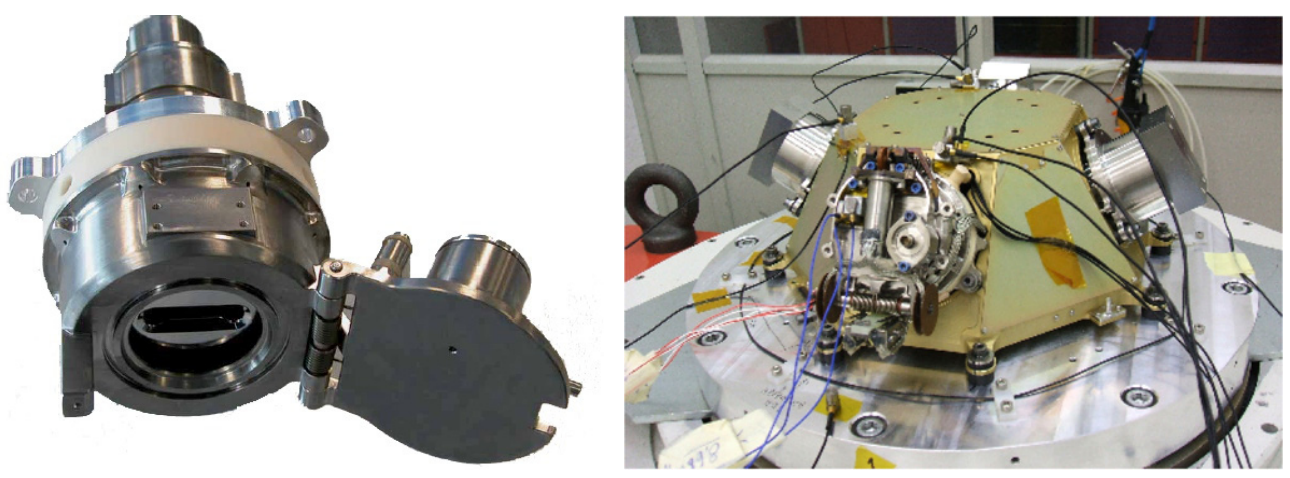

Figure 8. Slit FEEPs: detail of a slit FEEP prototype under testing (left) and the 4-FEEP assembly (right) forming one of the three thruster units to be mounted on the LPF hull.

$(0.1-150)$ with a resolution of $0.1 \mu \mathrm{N}$ and noise $\leqslant 0.1 \mu \mathrm{N} \mathrm{Hz}^{-1 / 2}$ (in the range from $10^{-4}$ to $1 \mathrm{~Hz}$ ). The micro-propulsion system of LPF is designed to avoid inducing magnetic or electric disturbances, gas leaks and mechanical noise. Its functioning principle is based on the extraction and acceleration of positive ions from a liquid metal reservoir by means of a very strong electric field (up to $10^{9} \mathrm{~V} \mathrm{~m}^{-1}$ ). A dedicated charge neutralizer balances the loss of positive ions with electrons extraction. Two FEEPs models are actually under study, based on slit (baseline, liquid caesium, see figure 8) and needle (back-up, liquid indium) technologies. In both cases, multi-axes motion is obtained by coupling FEEPs into clusters of four and mounting the final assemblies on the SC side hull at $120^{\circ}$ to one another. LPF will also carry sets of colloidal thrusters from NASA with the same resolution of ESA FEEPs but range upper limited to $30 \mu \mathrm{N}$.

\subsection{Diagnostics on-board}

Following the same philosophy of system identification that will allow us to single out contribution to acceleration by injection of dedicated electrostatic signals, thermal and magnetic signals will be induced by heaters and coils. Together with their sensing counterparts (thermometers and magnetometers), these pieces of hardware constitute part of the data diagnostic system [12], whose final component is a radiation monitor [17] that will be used as an independent tracer of the charging rate variability (one of the major noise sources for LISA).

\subsection{Orbit and lifetime}

The LPF final orbit will be a free Lissajous evolution around the Sun-Earth Lagrangian point L1, gravitating in the Sun field to mimic LISA's final orbit trailing the Earth. L1 provides a very stable environment in terms of thermal, gravity and electro-magnetic gradients and such a location will save efforts on the launcher, platform and communication scheme.

The size of the orbit will be $\sim 5 \times 10^{5} \mathrm{~km}$ by $8 \times 10^{5} \mathrm{~km}$ and the spacecraft will enter revolution around L1 after 15 apogee raising manoeuvers around Earth. LPF will be equipped with a dedicated engine to push it till injection on libration around L1. Launch date is at the moment foreseen for the end of 2010 from Kourou, French Guyana on-board a small launcher, likely of the new VEGA class. The science mission lifetime will nominally last 


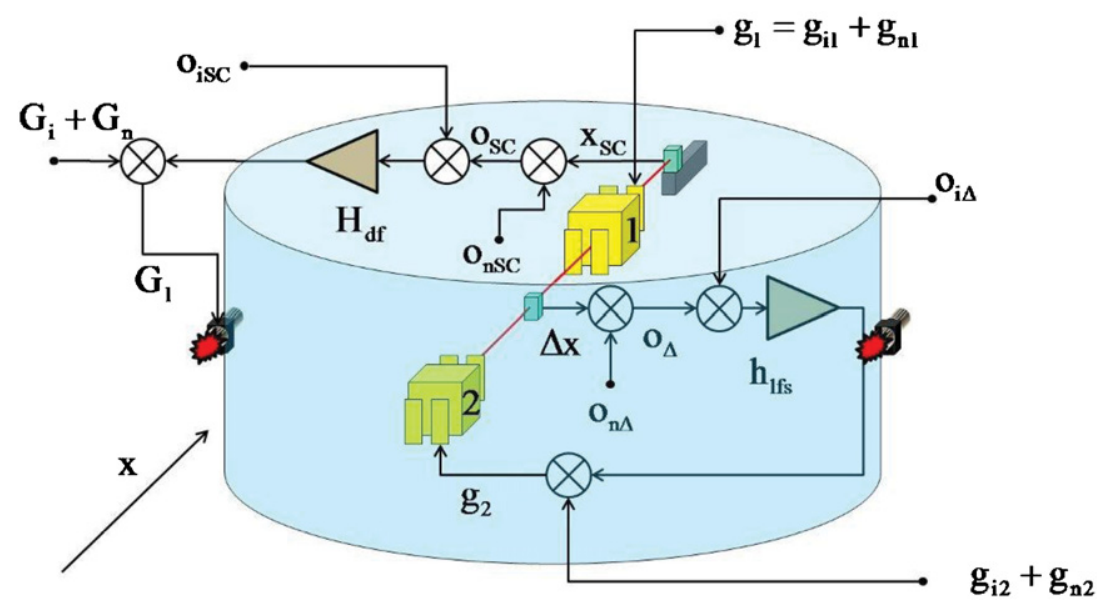

Figure 9. Schematic of the experiment. Two free-flying TMs (cubes 1 and 2) are included within a single SC (embedding cylinder). The (phase) signal $o_{\mathrm{SC}}$ of an interferometer reads out the displacement $x_{\mathrm{SC}}$ of the SC relative to TM1 along the sensitive axis $x$ superimposed to noise $o_{\mathrm{nSC}}$. The signal $o_{\Delta}$ of a second interferometer reads out the displacement $\Delta x$ of TM2 relative to TM1, added to noise $o_{\mathrm{n} \Delta}$. Forces are applied to both TMs via the GRS (plates flanking TM1 and TM2), and to the SC through the micro-thrusters (side plumes). A drag-free control loop, of gain $H_{\mathrm{df}}$ driven by $o_{\mathrm{SC}}$ and acting on thrusters, forces the spacecraft to follow TM1. A suspension loop of gain $h_{\mathrm{lfs}}$, driven by $o_{\Delta}$, applies an electrostatic force on TM2, forcing it to follow again TM1. Bias signals $o_{\mathrm{iSC}}$ and $o_{\mathrm{i} \Delta}$ can be injected in both loops. Command forces (per unit mass of each TM) $g_{\mathrm{i} 1}, g_{\mathrm{i} 2}$ and $G_{\mathrm{i}}$ can directly be applied to TMs and SC, respectively. Finally also noisy accelerations $g_{\mathrm{n} 1}, g_{\mathrm{n} 2}$ and $G_{\mathrm{n}}$ contribute to total accelerations $g_{1}, g_{2}$ and $G$ on TM1, TM2 and SC, respectively.

180 days including LTP operations, those dedicated to the NASA-provided disturbance reduction system [18], joint operations and commissioning phases.

\section{LISA Pathfinder and LISA as dynamical systems}

From a conceptual point of view, the LTP experiment is described by the scheme in figure 9, to which we refer for illustration and notation purposes.

The linearized, Newtonian equations for the dynamics of such a system may be written in the domain of the Laplace angular frequency $s$. Along the $x$-axis, thus:

$$
\begin{aligned}
& x_{\mathrm{SC}}\left(s^{2}+\omega_{1}^{2}\right)=G-g_{1}, \\
& \Delta x\left(s^{2}+\omega_{2}^{2}\right)-x_{\mathrm{SC}}\left(\omega_{2}^{2}-\omega_{1}^{2}\right)=g_{2}-g_{1} .
\end{aligned}
$$

The force gradients acting on TMs have been linearized as $\omega_{1}^{2} x_{\mathrm{SC}}$ for TM1 and $-\omega_{2}^{2}\left(\Delta x-x_{\mathrm{SC}}\right)$ for TM2, and written explicitly on the left-hand side. The 'stiffness' coefficients $\omega_{1}^{2}$ and $\omega_{2}^{2}$ are expected to be dominated by the electric and gravitational field gradients and to be negative. In (4), we have neglected the irrelevant terms proportional to the mass ratio between TMs and $\mathrm{SC}$, and to the direct gravitational coupling between the TMs.

Ideally the two interferometers independently measure $x_{\mathrm{SC}}$ and $\Delta x$. In this limit, one can then substitute signals in place of coordinates; in addition, one can write explicitly the forces due to the control loops. For the case where no force signal is purposely applied to the system, we get 


$$
\begin{aligned}
& \left(s^{2}+\omega_{1}^{2}+H_{\mathrm{df}}\right) o_{\mathrm{SC}}=G_{\mathrm{n}}-g_{\mathrm{n} 1}+\left(s^{2}+\omega_{1}^{2}\right) o_{\mathrm{nSC}} \\
& \left(s^{2}+\omega_{2}^{2}+h_{\mathrm{lfs}}\right) o_{\Delta}=g_{\mathrm{n} 2}-g_{\mathrm{n} 1}+\left(\omega_{2}^{2}-\omega_{1}^{2}\right)\left(o_{\mathrm{SC}}-o_{\mathrm{nSC}}\right)+\left(s^{2}+\omega_{2}^{2}\right) o_{\mathrm{n} \Delta} .
\end{aligned}
$$

Equations (5) may be compared with the formula for one LISA arm under the same idealized conditions, and in the absence of gravitational signal. Here the arm is formed by two TM (TM1 and TM2), contained in two different SC (SC1 and SC2), each chasing its own TM within a drag-free loop driven by the corresponding interferometer signals $o_{\mathrm{SC} 1}$ and $o_{\mathrm{SC} 2}$. We obtain

$$
\begin{aligned}
& \left(s^{2}+\omega_{1}^{2}+H_{\mathrm{df} 1}\right) o_{\mathrm{SC} 1}=G_{\mathrm{n} 1}-g_{\mathrm{n} 1}+\left(s^{2}+\omega_{1}^{2}\right) o_{\mathrm{nSC} 1}, \\
& \left(s^{2}+\omega_{2}^{2}+H_{\mathrm{df} 2}\right) o_{\mathrm{SC} 2}=G_{\mathrm{n} 2}-g_{\mathrm{n} 2}+\left(s^{2}+\omega_{2}^{2}\right) o_{\mathrm{nSC} 2}, \\
& s^{2} o_{\Delta}=g_{\mathrm{n} 2}-g_{\mathrm{n} 1}+\omega_{2}^{2}\left(o_{\mathrm{SC} 2}-o_{\mathrm{nSC} 2}\right)-\omega_{1}^{2}\left(o_{\mathrm{SC} 1}-o_{\mathrm{nSC} 1}\right)+s^{2} o_{\mathrm{n} \Delta} .
\end{aligned}
$$

The signal $o_{\Delta}$, measuring the TM1-to-TM2 displacement, is synthesized as $o_{\Delta}=$ $o_{\mathrm{SC} 1}+o_{\mathrm{SC}-\mathrm{SC}}-o_{\mathrm{SC} 2}$ with $o_{\mathrm{SC}-\mathrm{SC}}$ that from the SC-SC interferometer, with noise $o_{\mathrm{n} \Delta}$. The third line of (6) confirms the picture described in the introduction. The left-hand side is the frequency domain expression for the second derivative of the signal, which is equated to the difference of force per unit mass between the TM, i.e. the relative acceleration. This is expressed as a position-independent part $g_{\mathrm{n} 2}-g_{\mathrm{n} 1}$ plus the effect of the force gradient multiplying the jitter of each SC relative to its $\mathrm{TM}, \omega_{2}^{2}\left(o_{\mathrm{SC} 2}-o_{\mathrm{nSC} 2}\right)-\omega_{1}^{2}\left(o_{\mathrm{SC} 1}-o_{\mathrm{nSC} 1}\right)$. The acceleration signal is then added to the effect of the readout noise $s^{2} o_{\mathrm{n} \Delta}$, rapidly increasing with the frequency $|s|$.

The corresponding differential signal in LPF, shown in the second line of (5), is a close representation of the LISA case, except for two differences. First, because of the suspension loop, the acceleration signal must be obtained by a more complicated filtering of the signal $o_{\Delta}$ than just its second time derivative (we will show that this is not a limiting problem). Second, also in LPF part of the force, $\left(\omega_{2}^{2}-\omega_{1}^{2}\right)\left(o_{\mathrm{SC}}-o_{\mathrm{nSC}}\right)$ originates from the jitter of the SC relative to TM1. However here the jitter only produces a differential force if $\omega_{2}^{2} \neq \omega_{1}^{2}$ (consequences will be discussed later). Finally, let us remark that the dynamics of the local TM-SC link for LPF is nominally identical to that of LISA.

\section{Experiment approach}

Based on the analysis above, we now summarize the approach of the LTP experiment. As said, the first goal is to measure the power spectral density (PSD) of the total differential force noise on the TM, and to quantitatively compare it with a proper physical model, putting an upper limit to any unexplained source of noise, with an accuracy close to LISA requirements as in (3).

The experiment will thus prove the physical model to be used to calculate and confirm LISA sensitivity. A few constraints will be at play.

First the GRS is nominally identical to that of LISA. Most of the electrical disturbances, and any momentum exchange, acting on the surface of the TM are expected to be generated inside the GRS and to be at a level comparable to that of LISA. The rest of the disturbances however, mostly magnetic and gravitational, and those induced by the thermal fluctuation of the GRS interface, are system properties. LPF requirements foresee a slightly more relaxed control, to reduce costs and optimize the schedule. In particular requirements are only set for $f \geqslant 1 \mathrm{mHz}$, a graceful degradation being expected at lower frequency.

Second, as mentioned in LPF TM2 is forced to follow TM1 by the extra suspension loop (see figure 9) along the sensitive axis. The main function of the suspension is by all means to compensate for the difference of static gravitational force $F$ between the TMs that 
otherwise would quickly push TM2 out of its working position. This suspension loop is noisy as a consequence of the voltage fluctuation (a relative fluctuation of voltage $\delta V / V$ entails a relative force fluctuation of $2 \delta F / F)$. In addition, a force gradient is created-and thus a stiffness add-on—of order $2 F / d$ (where $d$ is the capacitor gap). As a result, a design trade-off was needed between the accuracy of balance of the gravitational force and the stability of the electronics generating the actuation voltage. The compromise sees an electronics stable at $2 \mathrm{ppm} \mathrm{Hz} \mathrm{Hz}^{-1 / 2}$ above $1 \mathrm{mHz}$, and an accuracy of static force cancellation of $\simeq 10^{-9} \mathrm{~m} \mathrm{~s}^{-2}$, a figure of the same order of that to be achieved in LISA, though for other reasons.

The gravitational balance is obtained by a proper set of compensation masses placed just outside the GRS. Unfortunately these also generate a quite substantial gravitational gradient, adding up to the excess gradient generated by the actuation voltage. As a consequence in LPF the tolerance towards force-per-unit-mass gradient is raised to $2 \times 10^{-6} \mathrm{~s}^{-2}$, one order of magnitude worse than LISA's $\left(4 \times 10^{-7} \mathrm{~s}^{-2}\right)$. This increased force gradient worsens the acceleration noise picture, despite the SC-TM jitter in LPF is the same as in LISA (same micro-thrusters and sensors within the drag-free loop). A partial mitigation comes in LPF as the effect multiplies the difference of stiffness between the TM, a number that is expected to be just twice the one predicted for LISA.

Adding up all the above, the total differential acceleration noise between the TMs is tolerated to be as high as seven times the LISA figure in (3) at frequencies $f \geqslant 1 \mathrm{mHz}$, with no requirements below this threshold. However this noise level is just the starting point of a detailed set of experimental procedures [1, 19], whose objective is to quantify the leading contributions to the total figure. To carry out this procedure, and not to be limited by the readout noise, LPF uses for both $o_{\mathrm{SC}}$ and $o_{\Delta}$ a heterodyne interferometer with the same performance required by all LISA interferometers, $6.5 \sqrt{1+(f / 3 \mathrm{mHz})^{-4}} \mathrm{pm} \mathrm{Hz}^{-1 / 2}$. This also makes the local SC-TM link a faithful representation of the LISA one in all respects, including its performance as a drag-free loop system.

The details of this experimental plan have been discussed in [1, 19]. In the following section, we expand some specific examples, namely:

- the measurement of total acceleration noise and the separation of the effect of SC jitter;

- the measurement of some key relevant dynamical parameters, in particular the stiffness coefficients, the gravitational gradient and the calibration of all force actuators;

- the measurement of the effect of the intermediate bodies motion (SC) in the TM-to-TM link;

- the measurement of dc gravitational force and the actuation noise.

\section{Measurement of acceleration noise and the separation of SC jitter}

Total acceleration noise, according to (5), can be measured by filtering the differential accelerometer output $o_{\Delta}$ with the filter [20] of transfer function $\left(s^{2}+\omega_{2}^{2}+h_{\mathrm{lfs}}(s)\right)$. The filter demands to apply a second derivative to the data and knowledge of both the stiffness and the real transfer function of the suspension control loop. This in particular requires knowledge of the absolute calibration of the electrostatic actuator, of the delays due to data transmission, etc. The second derivative can be approximated with a standard Finite Impulse Response filter with reasonable accuracy [21]. The measurement of stiffness and of the actual loop transfer functions will be described in detail in the following section. The overall transfer function can then be approximated with arbitrary precision by an auto-regressive moving average filter to be applied to the data. As high as the suspension loop gain is, the resulting filters do not involve time constants longer than $\sim 100 \mathrm{~s}$; hence, very short stretches of data will be sacrificed 

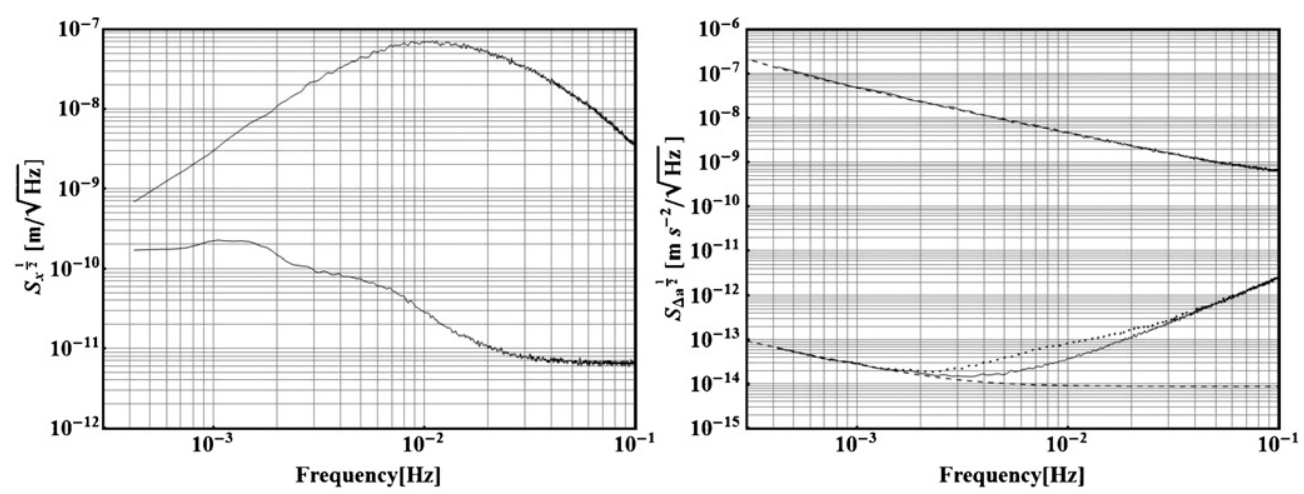

Figure 10. A simulated example of conversion of interferometer data to estimated acceleration. Left. Upper line: PSD of simulated data for $o_{\mathrm{SC}}$. Lower line: PSD for $o_{\Delta}$. Right. Upper continuous line: estimated PSD for $G_{\mathrm{n}}-g_{\mathrm{n} 1} \simeq G_{\mathrm{n}}$. Upper dashed line: input PSD for $G_{\mathrm{n}}-g_{\mathrm{n} 1}$ used in the simulation. Lower dotted line: total estimated differential acceleration PSD. Lower continuous line: estimated PSD for $g_{\mathrm{n} 2}-g_{\mathrm{n} 1}$. Lower dashed line: input PSD for $g_{\mathrm{n} 2}-g_{\mathrm{n} 1}$ used in the simulation. The simulation has been performed with a SC jitter slightly larger than requirements for illustration purposes.

in the conversion. An example of a simulated implementation of this procedure is shown in figure 10 .

A first example of experimental identification of a specific source of noise is the separation of the effect of SC jitter. Before we proceed with the discussion, it must be noted that the idealized picture in (5) does not take into account the presence of an intermediate body within the TM-to-TM link, namely the optical bench that, with good accuracy, is rigidly attached to the SC. Thus physically the link measures an inaccurate combination of $x_{\mathrm{SC}}$, the relative displacement between SC and TM1, and the relative displacement $x_{\mathrm{SC} 2}$ between SC and TM2. The differential interferometer signal is then $o_{\Delta}=\left(1+\delta_{\Delta \mathrm{SC}}\right) x_{\mathrm{SC}}-x_{\mathrm{SC} 2}$ and the effect of this cross-talk is the addition of an acceleration-like term $\delta_{\Delta \mathrm{SC}}\left(o_{\mathrm{SC}}-o_{\mathrm{nSC}}\right)\left(s^{2}+\omega_{2}^{2}\right)$ on the right-hand side of the second line of (5). If the coefficient $\delta_{\Delta \mathrm{SC}}$ has been measured then the following combination of signals [20]:

$$
\Delta a=\left(s^{2}+\omega_{2}^{2}+h_{\mathrm{lfs}}\right) o_{\Delta}-\left(\omega_{2}^{2}-\omega_{1}^{2}+\delta_{\Delta \mathrm{SC}}\left(s^{2}+\omega_{2}^{2}\right)\right) o_{\mathrm{SC}}
$$

is an estimate of $g_{\mathrm{n} 2}-g_{\mathrm{n} 1}$, corrupted by the readout noise:

$$
-\left(\omega_{2}^{2}-\omega_{1}^{2}+\delta_{\Delta \mathrm{SC}}\left(s^{2}+\omega_{2}^{2}\right)\right) o_{\mathrm{nSC}}+\left(s^{2}+\omega_{2}^{2}\right) o_{\mathrm{n} \Delta} \simeq o_{\mathrm{n} \Delta} s^{2} .
$$

For simulated data the result of such a data reduction procedure is illustrated in figure 10. It is worth noting that the subtraction of this noise source is made possible by the availability of a low-noise interferometric readout for $x_{\mathrm{SC}}$, otherwise the correction would bring about excess noise obscuring its own benefit. Note that this procedure can be extended to the LISA link as the SC to TM high-accuracy readout is available there too. This significantly reduces the concern for the effect of force gradients.

\section{The measurement of dynamical parameters}

The discussion in section 5 shows that knowledge of a few parameters is paramount to enable the projection of noise onto its basic constituent sources. In a simplified linear response model 
of the dynamics these parameters fall within a few broad categories:

- calibration of, and cross-talk among, available signals;

- calibration of, and cross-talk among, available force and torque actuators;

- matrix of coupling stiffness coefficients for SC and TM;

- static force vectors acting on SC and TMs.

All these parameters, except for static forces and torques, may also be frequency dependent. However, at the very low frequencies discussed here, the residual frequency dependence can reasonably be parameterized as barely linear.

In the linear equations (5), one may express the lack of knowledge of the parameters entering the dynamics by redefining them according to

$$
\begin{array}{llll}
\omega_{1}^{2} & \rightarrow \omega_{1}^{2}\left(1+\delta_{x 1}\right), & \omega_{2}^{2} \rightarrow \omega_{2}^{2}\left(1+\delta_{x 2}\right), \\
h_{\mathrm{lfs}} \rightarrow h_{\mathrm{lfs}}\left(1+\delta_{h}+\delta_{s h} \frac{s}{\omega_{0}}\right), & H_{\mathrm{df}} \rightarrow H_{\mathrm{df}}\left(1+\delta_{H}+\delta_{s H} \frac{s}{\omega_{0}}\right), \\
o_{\mathrm{SC}} \rightarrow\left(1+\delta_{\mathrm{SC}}\right) x_{\mathrm{SC}}+\delta_{\mathrm{SC} \Delta} \Delta x, & o_{\Delta} \rightarrow\left(1+\delta_{\Delta}\right) \Delta x+\delta_{\Delta \mathrm{SC}} x_{\mathrm{SC}}
\end{array}
$$

Here each parameter is substituted by its nominal value multiplied by a factor containing an uncertainty $\delta_{\#}$. In addition, the calibration of the suspension and drag-free loop gains, depending on the calibration of thrusters and electrostatic actuators respectively, also include a frequency-dependent term. To make uncertainty parameters non-dimensional, we have introduced the angular frequency $\omega_{0}$, that we take equal to the upper end of the MBW. Except for the loop gains, all other parameters are assumed, based on a physical analysis, to be frequency independent.

In order to measure these parameters, stimulus signals may be injected in all available inputs of figure 9. The preferred way of deriving the parameters' values is then to perform a maximum likelihood fit on the measured signals, in this case $o_{\mathrm{SC}}$ and $o_{\Delta}$. This fit is in general nonlinear as parameters may appear in the denominator of the calculated transfer functions. In order to analyse the effective measurability of the various $\delta_{\#}$, we have set up a method based on the linearization of the system response as a function of the parameters. If we name the signal vector $\vec{o}$ of components $o_{\mathrm{SC}}$ and $o_{\Delta}$, its deviation from the nominal value $\vec{o}_{0}$ is

$$
\overrightarrow{\delta o}(t) \equiv \vec{o}(t)-\vec{o}_{0}(t) \simeq \sum_{k=1}^{N_{\mathrm{p}}} \vec{o}_{k}(t) \delta_{k} .
$$

Here $\vec{o}_{0}$ is the nominal response the system would display if all parameters took their nominal values. We have numbered the parameters from 1 to $N_{\mathrm{p}}=10\left(\delta_{1} \equiv \delta_{x 1}, \delta_{2} \equiv \delta_{x 2}, \ldots\right)$ appearing in (9). The functions $\vec{o}_{i}$ result from

$$
\vec{o}_{i}(t)=\left.\frac{\partial \vec{o}(t)}{\partial \delta_{i}}\right|_{\delta_{k} \rightarrow 0, \forall k \neq i} \cdot
$$

Equation (10) opens the door to a classical optimal filter approach [22] for the estimation of the $\delta_{i}$ coefficients. In particular, one can show that the residual cross-covariance of the parameters is given by

$$
\sigma_{\delta_{i} \delta_{j}}=\left(\eta^{-1}\right)_{i j}
$$

where the $N_{\mathrm{p}} \times N_{\mathrm{p}}$ matrix $\eta$ is given by

$$
\eta_{k, m}=\frac{1}{2 \pi} \int_{-\infty}^{\infty} \sum_{\alpha, \beta=1}^{2} \frac{o_{k, \alpha}(\omega) o_{m, \beta}(\omega)}{S_{\alpha, \beta}(\omega)} \mathrm{d} \omega,
$$


Table 1. Coefficient values for the signal $o_{\mathrm{iSC}}$ and $o_{\mathrm{i} \Delta}$ injected into the drag-free and suspension control loop, respectively.

\begin{tabular}{lcclll}
\hline $\begin{array}{l}\text { Frequency } \\
(\mathrm{Hz})\end{array}$ & $\begin{array}{l}\text { Number of } \\
\text { cycles }\end{array}$ & $\begin{array}{l}\text { Start } \\
\text { time }(\mathrm{s})\end{array}$ & $\begin{array}{l}\text { Stop } \\
\text { time }(\mathrm{s})\end{array}$ & $\begin{array}{l}\text { Amplitude } \\
o_{\text {iSC }}(\mu \mathrm{m})\end{array}$ & $\begin{array}{l}\text { Amplitude } \\
o_{\mathrm{i} \Delta}(\mu \mathrm{m})\end{array}$ \\
\hline $8.33 \times 10^{-4}$ & 1 & 0 & 1200 & 1 & 0.80 \\
$1.67 \times 10^{-3}$ & 2 & 1300 & 2500 & 1 & 0.48 \\
$3.33 \times 10^{-3}$ & 4 & 2600 & 3800 & 1 & 0.19 \\
$6.67 \times 10^{-3}$ & 8 & 3900 & 5100 & 1 & 0.088 \\
$1.33 \times 10^{-2}$ & 16 & 5200 & 6400 & 0.58 & 0.096 \\
$2.67 \times 10^{-2}$ & 32 & 6500 & 7700 & 0.28 & 0.18 \\
$5.33 \times 10^{-2}$ & 64 & 7800 & 9000 & 0.14 & 0.46 \\
\hline
\end{tabular}

and the $o_{k, \alpha}, \alpha=1,2$ are the two component of the $\vec{o}_{k}$ vector in Fourier domain. $S_{\alpha, \beta}$ is the (cross-)PSD of the readout noise $o_{\mathrm{nSC}}$ and $o_{\mathrm{n} \Delta}$, organized in a vector $\vec{o}_{\mathrm{n}}$ of components $o_{\mathrm{n}, \alpha}, \alpha=1,2$.

We give here as an example the results of this procedure when applied to the parameters in (9). The system is stimulated with two independent signals $o_{\mathrm{iSC}}$ and $o_{\mathrm{i} \Delta}$ whose characteristics are shown in table 1 . The rationale behind the choice is that of a uniform scanning of the logarithm of the frequency approximately within the MBW. The time spent at each frequency is constant, two adjoining frequencies being separated by a $100 \mathrm{~s}$ stimulus-less lag to make data reduction easier. Amplitudes are chosen not to require more than $10 \%$ of the available actuation authority within the relevant low noise mode. In addition, input signals are designed not to push the TM outside its working volume of about $10 \mu \mathrm{m}$ side and $0.1 \mathrm{mrad}$ angular rotation.

The evoked response is not sufficient to disentangle the contributions due to all the $N_{\mathrm{p}}$ unknowns in (9). It turns out that excitation with the signal in $o_{\mathrm{iSC}}$ produces a cross correlation matrix in (12) with only six eigenvalues $<100$ (a reasonable upper limit for the square errors of parameters whose values should by construction be $<1$ ). Thus only six combinations of imperfections are in practice measurable with this method. Therefore, the rank $R$ of the matrix that converts the original imperfections to the independently measured ones is $R=6<N_{\mathrm{p}}=10$ : the relation is not invertible and the experiment is not sufficient, as expected, to give a significant measurement for all parameters in (9).

Adding an experiment where a signal is instead injected into $o_{\mathrm{i} \Delta}$, three new combination are measured with significant signal-to-noise ratio. This brings the number of independently measured combination to $9<N_{\mathrm{p}}=10$, still insufficient to derive all the $N_{\mathrm{p}}$ parameters. However the overall calibration for both interferometers, $\delta_{\mathrm{SC}}$ and $\delta_{\Delta}$ are measured on ground with accuracies better than $\sigma \simeq 10^{-4}$. This is also the case for the cross-talk from the relative displacement of TMs to $o_{\mathrm{SC}}$, a value that can be safely capped to $\left|\delta_{\mathrm{SC} \Delta}\right|<10^{-3}$. With these assumptions we get $R>N_{\mathrm{p}}$ and we can finally find the pseudo-inverse matrix corresponding to minimum uncertainties for the original parameters. These minimal uncertainties are listed in table 2. It must be noted however that some of the coefficients show strong correlations.

The combination of measurements shown allows us to fully calibrate the dynamics and in particular to measure the stiffness coefficients with sufficient relative accuracy to separate the role of gravitational gradient. This is expected to be $\geqslant 5 \%$ of the total stiffness on TM1 and thus in principle to be measurable with a relative accuracy of $2-3 \%$. 
Table 2. Residual uncertainty on parameter values.

\begin{tabular}{ll|ll}
\hline Parameter & Error & Parameter & Error \\
\hline$\delta_{H}$ & $3.6 \times 10^{-3}$ & $\delta_{s h}$ & $3.1 \times 10^{-5}$ \\
$\delta_{h}$ & $1.0 \times 10^{-4}$ & $\delta_{\Delta \mathrm{SC}}$ & $1.0 \times 10^{-7}$ \\
$\delta_{\mathrm{SC}}$ & $1.0 \times 10^{-4}$ & $\delta_{\Delta}$ & $1.0 \times 10^{-4}$ \\
$\delta_{\mathrm{SC} \Delta}$ & $1.0 \times 10^{-3}$ & $\delta_{x 1}$ & $1.2 \times 10^{-3}$ \\
$\delta_{s H}$ & $2.2 \times 10^{-3}$ & $\delta_{x 2}$ & $7.0 \times 10^{-4}$ \\
\hline
\end{tabular}

\section{Parabolic flight in $10^{-11} \mathrm{~g}$ and the projection of actuation noise}

As explained already, one limiting source of noise in LPF is the noise of the electrostatic actuation used to compensate the static difference of force between TMs, mostly of static gravitational nature. This force would accelerate TM2 relative to TM1 and SC and de-stabilize the system. This additional force is not needed in LISA and thus appears as an intrinsic limit of the LTP tests. To estimate and/or to subtract this noise contribution, at a very early stage of the project, it was proposed to let TM2 drift free of any control and actuation and measure the residual noise. Unfortunately the expected local static force along $x$, of order $F \simeq 2 \mathrm{nN}$ $\left(5 \times 10^{-11} \mathrm{~g}\right.$ per unit TM), is sufficient to move TM2 by $>0.5 \mathrm{~mm}$ in $1000 \mathrm{~s}$, bringing it well outside the range where the system is reasonably linear. Thus a noise measurement in one single stretch of free fly is highly unlikely to provide a noise measurement down to the $\mathrm{mHz}$ frequencies of interest here.

However a different mode of operation is being actively studied [23], where repeated impulses of force, of duration $T_{\mathrm{d}} \leqslant 1 \mathrm{~s}$, are applied to TM2 in contrast to the direction of the static force. This way TM2 undergoes repeated stretches of 'parabolic flight', starting from the farthest possible position of $x_{\min } \simeq-10 \mu \mathrm{m}$ and flying to $x_{\max } \simeq+10 \mu \mathrm{m}$ (around the nominal zero) before motion would invert under the effect of the static force and TM2 would start moving again towards the initial position (the positive $x$-direction is taken antiparallel to the static force). At frequencies well below the inverse of repetition period of the pulses, $\Delta T \simeq 200 \mathrm{~s}$, the force noise is in principle unaffected by the noisy pulse and its PSD can be estimated by just putting to zero the data within the short-time windows during which pulses are applied. The resulting parabolic flight can be fit to the proper dynamical model, thus also giving a measurement for the static force, and subtracted from the data prior PSD estimation, a procedure that induces a negligible negative bias to the spectrum computation.

The soundness of this procedure is confirmed by preliminary calculations. The remaining problems, currently under active investigation by the team, are with the practical implementation of a procedure that requires:

- A control loop to calculate the amplitude of force pulses to be given to TM2 when it falls back to $x_{\min }$ with partly random velocity. This loop must basically provide pulses of force amplitude $F_{\mathrm{p}}$ such that, on the average, $F_{\mathrm{p}} T_{\mathrm{p}} / \Delta T=F$. The related control law appears to be easily computable.

- During the pushes of actuation, the angular control around $z$ is perturbed by the high voltages producing the $x$-force pulses. An active suppression of the cross-talk between the $x$-force and $z$-torque was designed to reduce this, allowing for a smoother control of torque along $z$ during the drift/parabolic flight. 
Preliminary results [23] are encouraging. If confirmed, the method will allow the overcoming of this noise source and the achievement of an upper limit for non-modelled forces acting on LISA TMs appreciably closer to LISA requirement in (3) than the formal mission goal.

\section{Conclusions and summary}

In the effort of keeping the paper compact, we willingly skipped several details about the hardware [3], the measurement strategy [19] and the data analysis effort [24] underneath the progress shown. We nevertheless showed that at 2 years from launch the advance is adequate to the challenge and LPF is in every aspect on the way to fulfilling its duties as geodesics explorer and technology demonstrator for LISA.

\section{References}

[1] Anza S et al 2005 The LTP experiment on the LISA pathfinder mission Class. Quantum Grav. 22 S125-38

[2] McNamara P, Vitale S and Danzmann K 2008 Lisa pathfinder Class. Quantum Grav. 25114034

[3] Racca G and McNamara P 2009 The lisa pathfinder mission tracing Einstein's geodesics in space Space Sci. Rev. at press

[4] Synge J L 1964 Relativity: The General Theory (Amsterdam: North-Holland)

[5] Chauvineau B, Regimbau T, Vinet J Y and Pireaux S 2005 Relativistic analysis of the Lisa long range optical links Phys. Rev. D 72122003

[6] Bertotti B, Iess L and Tortora P 2003 A test of general relativity using radio links with the Cassini spacecraft Nature 425374

[7] Weber W J, Bortoluzzi D, Cavalleri A, Carbone L, Lio M Da, Dolesi R, Fontana G, Hoyle C D, Hueller M and Vitale S 2003 Position sensors for flight testing of LISA drag-free control Gravitational-Wave Detection Proc. SPIE vol 4856 ed M Cruise and P Saulson (Bellingham, WA: SPIE Optical Engineering Press) pp 31-42

[8] Whitcomb S E 2008 Ground-based gravitational-wave detection: now and future Class. Quantum Grav. 25114013

[9] Elliffe E J, Bogenstahl J, Deshpande A, Hough J, Killow C, Reid S, Robertson D, Rowan S, Ward H and Cagnoli G 2005 Hydroxide-catalysis bonding for stable optical systems for space Class. Quantum Grav. 22 S257-67

[10] Heinzel G et al 2006 Interferometry for the LISA technology package LTP: an update J. Phys. Conf. Ser. 32 132-6

[11] Ortega-Ruiz J A, Conchillo A, Xirgu X and Boatella C 2006 Mission critical software in LISA pathfinder AIP Conf. Proc. vol 873 ed S M Merkowitz and J C Livas (New York: AIP) pp 697-701

[12] Lobo A et al 2006 In-flight diagnostics in LISA pathfinder AIP Conf. Proc. vol 873 ed S M Merkowitz and J C Livas (New York: AIP) pp 522-8

[13] Fichter W, Schleicher A and Vitale S 2008 Drag-free control design with cubic test masses Lasers, Clocks and Drag-Free Control: Exploration of Relativistic Gravity in Space (Astrophysics and Space Science Library vol 349) ed H Dittus, C Lämmerzahl and S G Turyshev p 361

[14] Fichter W, Gath P, Vitale S and Bortoluzzi D 2005 LISA pathfinder drag-free control and system implications Class. Quantum Grav. 22 S139-48

[15] Fichter W, Schleicher A, Bennani S and Wu S 2007 Closed loop performance and limitations of the LISA pathfinder drag-free control system AIAA Guidance Navigation and Control Conference (South Carolina: Hilton Head) pp 20-3

[16] Shaul D N A, Araújo H M, Rochester G K, Schulte M, Sumner T J, Trenkel C and Wass P 2008 Charge Management for LISA and LISA Pathfinder Int. J. Mod. Phys. D 17 993-1003

[17] Wass P J, Araújo H, Boatella C, Chmeissani M, Hajdas W, Lobo A, Puigdengoles C and Sumner T 2006 The LISA pathfinder radiation monitor AIP Conf. Proc. vol 873 ed S M Merkowitz and J C Livas (New York: AIP) pp 225-9

[18] Folkner W M et al 2003 Disturbance reduction system: testing technology for precision formation control Proc. SPIE vol 4860 ed A B Schultz and R G Lyon (Bellingham, WA: SPIE Optical Engineering Press) pp 221-8

[19] Danzmann K et al 2007 Einstein's geodesic explorer: the science case for LISA pathfinder ESA-SCI 1 
[20] Vitale S 2007 Measurement of differential acceleration noise on LISA pathfinder Technical Report S2-UTNTN-3053, UNITN

[21] Carbone L, Ciani G, Dolesi R, Hueller M, Tombolato D, Vitale S, Weber W J and Cavalleri A 2007 Upper limits to surface-force disturbances on LISA proof masses and the possibility of observing galactic binaries Phys. Rev. D 75042001

[22] Papoulis A and Pillai S U 2002 Probability, Random Variables and Stochastic Processes 4th edn (New York: McGraw-Hill)

[23] Grynagier A, Fichter W and Vitale S 2009 The drift mode for LISA pathfinder: parabolic drag-free flight, actuation with kicks, spectral analysis with gaps Space Sci. Rev. at press

[24] Hewitson M et al 2009 Data analysis for the LISA technology package Class. Quantum Grav. 26094003 